\title{
Decreased IRF7 Expression Contributes to Deficient IFN- $\alpha$ Production of Plasmacytoid Dendritic Cells in Chronic HIV-1 Infected Men who have Sex with Men
}

\section{Wenqing Geng, Sha Li, Xuejie Fan, Fang Gao, Hualu Cui, Hong Sun, Zining Zhang, Yongjun Jiang and Hong Shang*}

Key Laboratory of AIDS Immunology of Ministry of Health, First Hospital of China Medical University, Shenyang, China

\section{Abstract}

Background: Deficient interferon (IFN)- $\alpha$ production of plasmacytoid DCs (pDCs) from HIV-1-infected individuals in response to virus or toll-like receptor (TLR) stimulation has been reported. pDCs express TLR7 and TLR9, but whether and how the TLR pathway contributes to the deficiency has not been well addressed.

Methods: Twenty-six subjects with HIV-1 infection were recruited from a chronic HIV-1 infection (CHI) cohort of men who have sex with men (MSM) and $15 \mathrm{HIV}-1$ negative healthy MSM were used as normal controls (NCs). Maturation markers (CD80, CD86, CD83, CD40, CCR7), TLR7 and TLR9, nuclear factor KB (NF-KB) and interferon regulatory factor 7 (IRF7) expression were detected using surface and intracellular multicolor flow cytometry. Intracellular IFN- $\alpha$ production of pDCs was measured in response to R848 (TLR7 agonist) and ODN2216 (TLR9 agonist), respectively.

Results: IFN- $\alpha$ production of $\mathrm{pDC}$ s with treatment of R848, but not ODN2216, from MSM with CHI was significantly lower than that of NCs. There was no significant difference in maturation marker expression between MSM with $\mathrm{CHI}$ and NCs. The expression of TLR7 and TLR9 on pDCs of MSM with $\mathrm{CHI}$ was significantly up-regulated. The expression of IRF7, a downstream signaling protein in the TLR7/9 signaling pathway, was significantly down-regulated as compared with that of NCs. NF-kB expression of pDCs of MSM with $\mathrm{CHI}$ were similar to that of NCs.

Conclusions: These results indicated that decreased IRF7 expression might contribute to deficient IFN- $\alpha$ production in MSM with $\mathrm{CHI}$, suggesting efforts to increase the expression of IRF7 might resume the function of pDCs in individuals with $\mathrm{CHI}$.

Keywords: HIV-1; Plasmacytoid dendritic cells; IFN-alpha; Toll-like receptor; IRF7

Abbreviation: HIV-1: Human Immunodeficiency Virus Type 1; CHI: Chronic HIV-1 Infection; PDCs: Plasmacytoid Dendritic Cells; IFN- $\alpha$ : Interferon- $\alpha$; TLR: Toll-like Receptor; TLR7: Toll-like Receptor 7; TLR9: Toll-like receptor 9; IRF7: Interferon Regulatory Factor 7; NF- $\kappa$ B: Nuclear factor $\kappa B$; NCs: Normal Controls; MSM: Men Who Have Sex With Man; PBMC: Peripheral Blood Mononuclear Cells; ICS: Intracellular Staining; SEM: Standard Error of the Mean

\section{Introduction}

Plasmacytoid dendritic cell (pDC), a subset of dendritic cells, is the major interferon (IFN)- $\alpha$ producing population and acts as sentinels for viral infection [1,2]. It was reported that IFN- $\alpha$ production of pDC correlated inversely with HIV-1 viral loads and were prognostic for the onset of opportunistic infections (OI) [3-7]. HIV-1 infectedpatients with depleted $\mathrm{CD} 4^{+} \mathrm{T}$ cell counts but in normal IFN- $\alpha$ response remained free of these OI [4]. Even though IFN-stimulated genes are reported to be up-regulated in vivo in chronic HIV-1 infection [8], in vitro stimulation by TLR7 or TLR9 ligands results in lower levels of IFN- $\alpha$ production in individuals with chronic HIV-1 infection (CHI) as compared to that in normal controls [9-13]. However, elite controllers and long-term non-progressors showed elevated IFN- $\alpha$ production [14]. These findings suggested an important role for IFN- $\alpha^{+} p D C$ in HIV-1 infection, but the underlying mechanism for the functional deficiency of pDC in $\mathrm{CHI}$ is still less clear.

In the present paper, we chose to study the phenotypic markers and TLR7/9 signaling pathways of pDCs in men who have sex with man (MSM) with CHI. Our study suggests that the decreased IFN- $\alpha$ production of pDCs after TLR7 stimulation may be due to the downregulated expression of IRF7.

\section{Materials and Methods}

\section{Subjects}

Twenty-six MSM with CHI were enrolled from the HIV-1-infected MSM cohort established by China Medical University and several provincial Centers for Disease Control and Prevention. These subjects have been infected for more than two years. None of the study subjects were under highly active antiretroviral therapy or co-infected with the hepatitis $\mathrm{B}$ or $\mathrm{C}$ virus before sample collection. Fifteen age-matched, healthy and HIV-1-negative MSM were selected randomly from an MSM cohort in Liaoning Province, Northeast China [15] and were used as normal controls (NCs) (Table 1). None of the total subjects self-reported injection drug use. Three cases (11.5\%) out of 26 HIV-1-infected MSM had self-reporting history of the rush poppers use, and two cases (13.3\%) did among NCs.

$\mathrm{CD}^{+} \mathrm{T}$ cell counts were measured using a FACSCalibur flow cytometer (BD Bioscience, San Jose, CA, USA). Plasma HIV-1 viral loads were determined by using a standardized RT-PCR assay (COBAS

*Corresponding author: Hong Shang, Director, Key Laboratory of AIDS Immunology of Ministry of Health, Department of Laboratory Medicine, First Hospital of China Medical University, Address: No. 155, Nanjingbei Street, Heping District, Shenyang, Liaoning province, 110001, China, Tel: 86-24-83282634; E-mail: hongshang100@hotmail.com

Received December 01, 2015; Accepted February 22, 2016; Published February 27, 2016

Citation: Geng W, Li S, Fan X, Gao F, Cui H, et al. (2016) Decreased IRF7 Expression Contributes to Deficient IFN- $\alpha$ Production of Plasmacytoid Dendritic Cells in Chronic HIV-1 Infected Men who have Sex with Men. J AIDS Clin Res 7: 548. doi:10.4172/2155-6113.1000548

Copyright: $\odot 2016$ Geng W, et al. This is an open-access article distributed under the terms of the Creative Commons Attribution License, which permits unrestricted use, distribution, and reproduction in any medium, provided the original author and source are credited. 
Citation: Geng W, Li S, Fan X, Gao F, Cui H, et al. (2016) Decreased IRF7 Expression Contributes to Deficient IFN- $\alpha$ Production of Plasmacytoid Dendritic Cells in Chronic HIV-1 Infected Men who have Sex with Men. J AIDS Clin Res 7: 548. doi:10.4172/2155-6113.1000548

Page 2 of 4

\begin{tabular}{|l|c|c|}
\hline Variable & CHI $(\mathbf{n = 2 6})$ & NCs $(\mathbf{n = 1 5})$ \\
\hline Age, years, median (IQR) & $30(26.0-33.25)$ & $25(23.75-31.75)$ \\
\hline HIV-1 subtype of A/E & $23(88.5 \%)$ & NA \\
\hline Estimated infection time, years, median (IQR) & $4(3-6)$ & NA \\
\hline CD4+ T-cell counts, cells/ $\mu$, median (IQR) & $417.5(357-606)$ & $951(780.5-1150.25)$ \\
\hline HIV-1 RNA load, Ig copies/ml, median (IQR) & $4.26(3.86-4.58)$ & NA \\
\hline
\end{tabular}

Data are no. (\%) of subjects, unless otherwise indicated.

CHI: Chronic HIV-1 Infection; NCs: Normal Controls; IQR: Interquartile Range; NA: Not Applicable.

Table 1: Characteristics of subjects with $\mathrm{CHI}$ and HIV-1 negative controls.

Amplicor, HIV-1 Monitor Test Version 2.0, Roche Diagnostics). The detection range was $40-10^{7}$ copies $/ \mathrm{ml}$ and the HIV-1 load less than the lower limit of detection was considered to be 40 copies/ml. Peripheral blood mononuclear cells (PBMCs) isolated from EDTA-coagulated blood of subjects were stored in liquid nitrogen. The study protocols and informed consent forms were approved by the Ethical Review Board of the First Hospital of China Medical University and the informed consent was obtained from all study subjects.

\section{Flow cytometry analysis}

The fluorescent monoclonal antibodies (mAbs) of lineage1 (CD3, CD14, CD16, CD19, CD20, CD56)-FITC, CD40-APC, CD83-PE, CCR7-

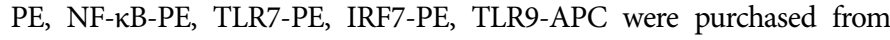
Becton Dickinson (BD), USA. The fluorescent mAbs of HLA-DR-APCCY7, CD123-PE-CY7, CD80-APC, and CD86-PE were obtained from BioLegend, USA. IFN- $\alpha$-APC was obtained from Miltenyi Biotec, Germany IgGl or IgG2a isotype was included in the assays as negative controls. At least 200,000 events were acquired for each sample and rare event analysis was conducted by on an LSRII cytometer (BD company, USA) using FACSDiva software. The region P1 was drawn to include lymphocytes and monocytes, excluding granulocytes (Supplementary Figure 1). The region P2 was gated tightly on lineage $1^{-} / \mathrm{HLA}-\mathrm{DR}^{+}$cells and the $\mathrm{CD} 123^{+}$population in $\mathrm{P} 3$ region was identified as pDCs. Gated from the region $\mathrm{P} 3$, the region $\mathrm{P} 4$ was $\mathrm{CD} 83^{+}$,

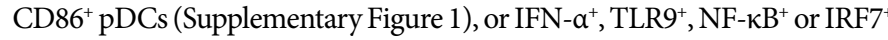
pDCs (Supplementary Figure 2). The region P5 gated from the region P3 was $\mathrm{CD} 40^{+}, \mathrm{CD}^{+} 0^{+}$, or $\mathrm{CCR}^{+}$pDCs (Supplementary Figure 1), or TLR7 ${ }^{+}$ (Supplementary Figure 2).
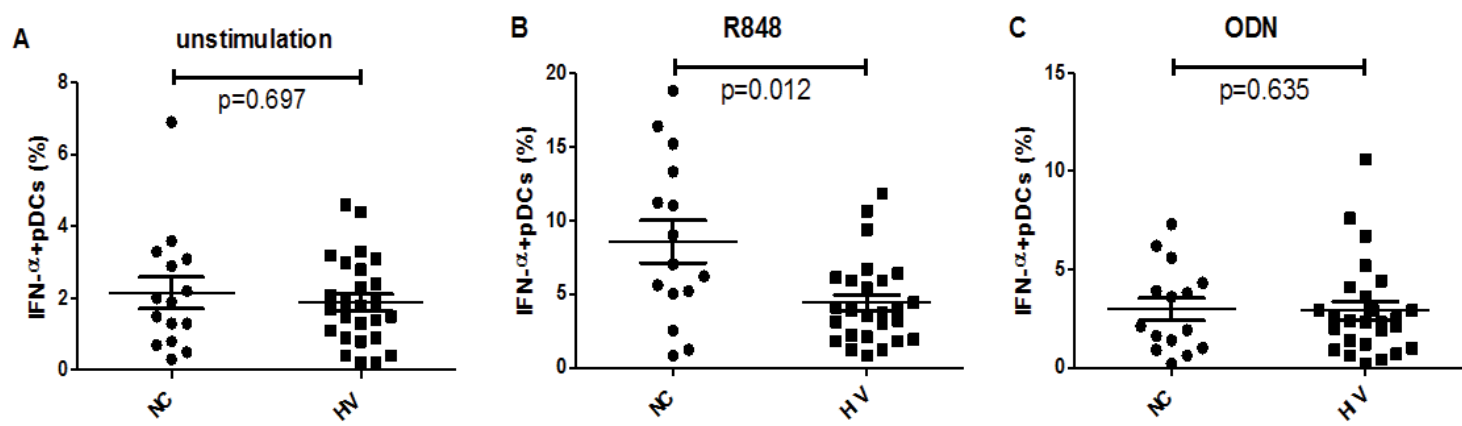

Figure 1: Reduced IFN- $\alpha$ production of pDCs stimulated with R848 in subjects with $\mathrm{CHI}$ as compared with NCs.

Significant decrease in IFN- $\alpha$ production was observed in pDCs stimulated with TLR7 agonist (R848) from individuals with $\mathrm{CHI}$ as compared with in NCs ( $p=0.012$ ), while there was no significant difference between $\mathrm{CHI}$ subjects and NCs when TLR9 agonist (ODN2216) was used ( $p=0.635)$. We also measured IFN- $\alpha$ production without stimulation and found no statistical differences between $\mathrm{CHI}$ subjects and NCs $(p=0.697)$. The data was displayed as mean with SEM.
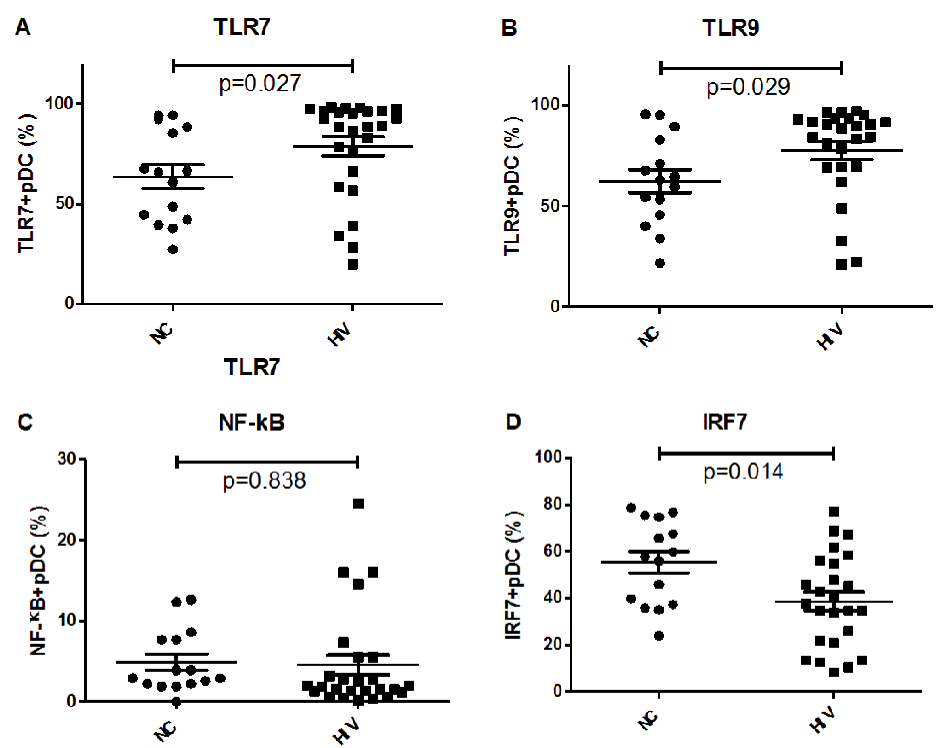

Figure 2: Increased TLR7 and TLR9 and decreased IRF7 expression of pDCs in subjects with CHI.

pDCs in individuals with chronic HIV-1 infection expressed higher levels of TLR7 and TLR9 than did normal controls ( $p=0.027$ and $p=0.031$, respectively) (Fig. 2 A and $\mathrm{B})$. The downstream signaling component IRF7 was significantly down-regulated as compared to normal controls ( $p=0.014)$ (Fig. 2D). However, expressions of NF-KB were similar between the HIV-1-infected subjects and the normal controls $(p=0.838)$ (Fig. 2C). The data was displayed as mean with SEM 


\section{Surface and intracellular staining of pDCs}

Cryopreserved PBMCs from subjects were thawed at $37^{\circ} \mathrm{C}$ washed twice, and resuspended in media before staining. To detect the expression of CD80, CD86, CD83, CD40 and CCR7 on the surface of pDCs, the following fluorescent $\mathrm{mAb}$ combinations were used: Lin-1/ HLA-DR/CD123/CD40/CD83, Lin-1/HLA-DR/CD123/CD80/CD86, and Lin-1/HLA-DR/CD123/CCR7.

For intracellular staining (ICS), PBMCs stained with lineage1, HLA-DR and CD123 was permeabilized with PermBuffer I (BD Biosciences) for 20 minutes at $4^{\circ} \mathrm{C}$. The cells were then stained with TLR7, TLR9, NF- $\kappa B$, IRF7 and IFN- $\alpha$ for 30 minutes at $4^{\circ} \mathrm{C}$, fixed and analyzed by flow cytometry. For measurement of IFN- $\alpha$ production in response to TLR stimulation, $2 \times 10^{6} \mathrm{PBMCs} / \mathrm{mL}$ were treated with 10 $\mu \mathrm{M}$ R848 (Invivogen, USA) [1] or $5 \mu \mathrm{M}$ ODN2216 (Invivogen, USA) for 6 hours in the presence of brefeldin A (BFA, BD, USA).

\section{Statistical analysis}

Data were analyzed by using GraphPad Prism 5 software (GraphPad Software, La Jolla, CA). Independent t test were used to analyze normally distributed continuous variables between two groups. Mann-Whitney $\mathrm{U}$ test was used for analysis of non-normally distributed continuous variables. A $p$ value $<0.05$ were considered to be statistically significant.

\section{Results}

\section{Deficient IFN- $\alpha$ production of $\mathrm{pDC}$ in subjects with $\mathrm{CHI}$ in response to TLR7 but not TLR9 stimulation}

Cryopreserved PBMCs from MSM with $\mathrm{CHI}$ and NCs were stimulated using ligands of TLR7 (R848) and TLR9 (ODN2216), respectively. The production of IFN- $\alpha$ in response to TLR7 agonist stimulation was compared between MSM with CHI and NCs. We found that the frequencies of IFN $-\alpha^{+}$pDCs in stimulation of TLR7 with R848 were significantly decreased in chronically infected MSM (mean 4.38\%; SEM, 0.56\%) as compared to that in NCs (mean, 8.56\%; SEM, $1.44 \%)(p=0.012)$. Following TLR9 stimulation with ODN2216, IFN- $\alpha$ production of pDCs in CHI was normal (mean, 2.91\%; SEM, 0.47\%) as compared to that in NCs (mean, 2.96\%; SEM, 0.56\%) $(p=0.635)$. We also measured the IFN- $\alpha$ production of unstimulated pDCs. We observed very low level of IFN- $\alpha^{+}$pDCs in the absence of simulation and no statistical differences between the HIV-1-infected MSM and NCs $(p=0.697)$ (Figure 1).

\section{No difference between phenotypic markers of pDCs in individuals with $\mathrm{CHI}$ and NCs}

Since the phenotypic status also affected the IFN- $\alpha$ production of pDCs [10], the activation markers on pDCs were analyzed. We found no difference between the expressions of CD40 in chronically HIV-1 infected individuals and normal controls $(p>0.05)$, and there was no significant correlation between CD40 expression and IFN- $\alpha$ production following TLR7 or TLR9 stimulation ( $p>0.05)$. Expressions of CD80, CD83, CD86, CCR7 on pDCs were similar in individuals with CHI and normal controls $(p>0.05)$. We find no significant correlation between IFN- $\alpha$ production and the expression of phenotypic markers (Table 2).

\section{Up-regulated TLR7 and TLR9 expression of pDCs in CHI}

As TLR7 and TLR9, expressed by pDC, play an important part on pDC function [16], we evaluated expressions of TLR7 and TLR9 to see whether their expression could regulate the deficient IFN-a production in our study population. The $\mathrm{pDCs}$ in individuals with $\mathrm{CHI}$

\begin{tabular}{|l|c|c|c|}
\hline Variables & CHI & NCs & P value \\
\hline CD40+pDCs\%, median (IQR) & $2.7(1.25-10.37)$ & $3.7(0.72-19.05)$ & 0.77 \\
\hline CD80+pDCs\%, median (IQR) & $7.15(1.77-14.87)$ & $9.75(5.42-20.15)$ & 0.75 \\
\hline CD83+pDCs\%, median (IQR) & $1.4(0.72-9.6)$ & $0.7(0.35-2.22)$ & 0.17 \\
\hline CD86+pDCs\%, median (IQR) & $0.45(0-1.6)$ & $0.3(0-1.15)$ & 0.58 \\
\hline CCR7+pDCs\%, median (IQR) & $0.55(0-0.97)$ & $0.85(0-2.45)$ & 0.42 \\
\hline
\end{tabular}

IQR: Inter Quartile Range. Use the Spearman rank correlation coefficient method. Table 2: Comparison of maturation marker expression on pDCs between MSM with $\mathrm{CHI}$ and $\mathrm{NCs}$.

\begin{tabular}{|l|c|c|c|c|}
\hline \multirow{2}{*}{ Variables } & \multicolumn{2}{|c|}{ IFN- $\boldsymbol{\alpha}+\mathbf{p D C} \% \mathbf{~ ( R 8 4 8 )}$} & \multicolumn{2}{c|}{ IFN- $\boldsymbol{\alpha}+\mathbf{p D C s} \%$ (ODN2216) } \\
\cline { 2 - 5 } & $\mathbf{r}$ & $\boldsymbol{p}$ value & $\mathbf{r}$ & $\boldsymbol{p}$ value \\
\hline CD40+pDCs\% & -0.42 & 0.08 & 0.17 & 0.48 \\
\hline CD80+pDCs\% & -0.41 & 0.09 & 0.29 & 0.24 \\
\hline CD83+pDCs\% & -0.30 & 0.23 & 0.36 & 0.14 \\
\hline CD86+pDCs\% & 0.00 & 0.99 & -0.01 & 0.96 \\
\hline CCR7+pDCs\% & -0.08 & 0.74 & 0.12 & 0.64 \\
\hline
\end{tabular}

Table 3: Correlation of maturation marker expression with IFN- $\alpha$ production of pDC induced by TLR7 and TLR 9 agonists in MSM with

expressed higher levels of TLR7 (mean, 78.87\%; SEM, 4.76\%) and TLR9 (mean, 77.44\%; SEM, 4.43\%) than did normal controls (mean, 63.79\%; SEM, 5.96\% versus mean, $62.47 \%$; SEM, $5.69 \% ; p=0.027$ and $p=0.029$, respectively) (Figure $2 \mathrm{~A}$ and $2 \mathrm{~B}$ ). Our above observations that similar and low IFN-a production in unstimulated $\mathrm{pDC}$ from controls and HIV infected groups (Figure 1A) as well as upregulated TLR 7 and 9 expressions in HIV infected group (Figure 2A and 2B) led us to further examine the downstream signaling proteins IRF7 and NF- $\kappa B$, which are regarded to directly regulate IFN- $\alpha$ production [17].

\section{Down-regulated IRF7 expression and similar NF-kB expression of pDCs in $\mathrm{CHI}$}

Since TLR7 and TLR9 expression seemed not to be responsible for deficiencies in IFN- $\alpha$ production, we further examined the downstream signaling proteins IRF7 and NF- $\kappa \mathrm{B}$, which are regarded to directly regulate IFN- $\alpha$ production [18].

We found that IRF7 ${ }^{+} \mathrm{pDC} \%$ was significantly decreased in individuals with $\mathrm{CHI}$ (mean, 38.72\%; SEM, 3.96\%) as compared to NCs (mean, 55.32\%; SEM, 4.63\%) ( $p=0.014$ ) (Figure 2D), while the NF- $k B$ expression was similar to NCs (mean, $4.56 \%$; SEM, $1.21 \%$ verse mean, 4.88; SEM, $1.01 \%$ ) ( $p=0.838$ ) (Figure $2 \mathrm{C}$ ). This result suggests that decreased IRF7 expression may lead to deficient IFN- $\alpha$ production of pDCs in individuals with CHI (Table 3).

\section{Discussion}

The recognition of pathogens by human pDCs is mediated primarily through recognition by TLR7 and TLR9, which trigger IFN- $\alpha$ production in response to infection by a variety of RNA and DNA viruses [19]. A major pathway of HIV-1 stimulation of IFN-a production by pDCs is through recognition of viral RNA by TLR7 $[20,21]$. It is likely that TLR7, rather than TLR9, mediates detection of HIV-1 and subsequent activation of pDCs as suggested by competition assays of TLR7 or TLR9 transfected cell lines [22]. Additionally, elevated levels of TLR7 in HIV-1-treated pDCs suggest a potential role for this receptor in the detection of HIV-1 [16]. Our findings were similar to others $[11,12]$ and confirmed further that, in chronic HIV-1 infection, IFN-a production following TLR7 stimulation was reduced.

Previous studies have shown that decreased TLR expression and function can account for increased susceptibility to infection and 
Citation: Geng W, Li S, Fan X, Gao F, Cui H, et al. (2016) Decreased IRF7 Expression Contributes to Deficient IFN- $\alpha$ Production of Plasmacytoid Dendritic Cells in Chronic HIV-1 Infected Men who have Sex with Men. J AIDS Clin Res 7: 548. doi:10.4172/2155-6113.1000548

deficiencies in the adaptive immune response [17,23,24]. Recent research on hepatitis $B$ virus also found that down-regulation of TLR7/9 leads to deficient production of IFN- $\alpha$ from pDCs [25]. However, in our study, we found that TLR7 and TLR9 levels were up-regulated rather than down-regulated in individuals with chronic HIV-1 infection, indicating that deficient IFN- $\alpha$ production following TLR7 stimulation was not due to the insufficient expression of TLR7 or TLR9.

Importantly, our present study observed decreased IRF7 expression in MSM with CHI as compare to that of NCs, while the expression levels of activation markers and NF- $\mathrm{KB}$ were similar to those of NCs. Tilton et al found that reduced IFN- $\alpha$ production in vitro was not due to diminished pDC numbers or trafficking to lymph nodes, but rather was due to feedback inhibition or prior stimulation through TLRs in vivo [13]. A recent study showed that diminished TLR7-induced IFN-a production by pDCs in HIV-1-infected individuals was not a consequence of downmodulation of the immune inhibitory receptor, BDCA- 2 on the $\mathrm{pDC}$ [20]. Taken together, we deduced that decreased IRF7 expression might lead to deficient IFN- $\alpha$ production of pDCs in individuals with CHI. It is of significance to provide a clue to discover potential molecular target for modulation in order to prevent immunopathology in HIV-1 infection. Additional, other molecules in the TLR7/9 signaling pathway might participate in the modulation of IRF7 since the up-regulation of TLR7/9 were observed in our present study and a negative feedback between TLR7/9 and IRF7 was reported by Honda [26].

It should be pointed out that the data presented herein are somewhat preliminary and a large-scale analysis is required in the near future. On the other hand, all subjects with CHI investigated in the present study were MSMs. Whether our present findings are applicable to the populations infected through other routes needs to be confirmed before getting solid conclusions. Nevertheless, this study would be helpful in the prevention and control of HIV-1 infection in China since the rapid increase of HIV-1 prevalence among Chinese MSM is of particular concern [18].

In summary, IRF7 seems to play a critical role in HIV-1-induced IFN- $\alpha$ production in human pDCs. Elucidating the mechanism by which IFN- $\alpha$ is regulated will help in understanding the role of pDCs in the immune response and in uncovering how IFN- $\alpha$ may be used as a treatment for HIV-1 infection.

\section{Financial Support}

This study was supported by grants from the Twelfth Five-Year Project on Tackling Key Problems of National Science and Technology (2012ZX10001-006), the National Natural Science Foundation of China (No. 81273238).

\section{Acknowledgements}

We acknowledge the staff of the local CDCs, who spent many hours working with us in collecting and verifying the data used in this study.

\section{References}

1. Ito T, Amakawa R, Kaisho T, Hemmi H, Tajima K, et al. (2002) Interferon-alpha and interleukin-12 are induced differentially by Toll-like receptor 7 ligands in human blood dendritic cell subsets. J Exp Med 195: 1507-1512.

2. Liu YJ (2005) IPC: professional type 1 interferon-producing cells and plasmacytoid dendritic cell precursors. Annu Rev Immunol 23: 275-306.

3. Sabado RL, O'Brien M, Subedi A, Qin L, Hu N, et al. (2010) Evidence of dysregulation of dendritic cells in primary HIV infection. Blood 116: 3839-3852.

4. Finke JS, Shodell M, Shah K, Siegal FP, Steinman RM (2004) Dendritic cell numbers in the blood of HIV-1 infected patients before and after changes in antiretroviral therapy. J Clin Immunol 24: 647-652.

5. Donaghy H, Pozniak A, Gazzard B, Qazi N, Gilmour J, et al. (2001) Loss of blood CD11c(+) myeloid and CD11c(-) plasmacytoid dendritic cells in patients with HIV-1 infection correlates with HIV-1 RNA virus load. Blood 98: 2574-2576.
6. Pacanowski J, Kahi S, Baillet M, Lebon P, Deveau C, et al. (2001) Reduced blood CD123+ (lymphoid) and CD11c+ (myeloid) dendritic cell numbers in primary HIV-1 infection. Blood 98: 3016-3021.

7. Geng W, Fan X, Diao Y, Cui H, Sun H, et al. (2011) Rapid disease progression in HIV-1-infected men who have sex with men is negatively correlated with peripheral plasmacytoid dendritic cell counts at the early stage of primary infection. J Clinlmmunol 31: 882-890.

8. Lehmann C, Harper JM, Taubert D, Hartmann P, Fatkenheuer G, et al. (2008) Increased interferon alpha expression in circulating plasmacytoid dendritic cells of HIV-1-infected patients. J Acquir Immune Defic Syndr 48: 522-530.

9. Chang JJ, Lacas A, Lindsay RJ, Doyle EH, Axten KL, et al. (2012) Differentia regulation of toll-like receptor pathways in acute and chronic HIV-1 infection. AIDS 26: 533-541.

10. Donhauser N, Pritschet K, Helm M, Harrer T, Schuster P, et al. (2012) Chronic immune activation in HIV-1 infection contributes to reduced interferon alpha production via enhanced CD40:CD40 ligand interaction. PLoS One 7: e33925.

11. Martinson JA, Roman-Gonzalez A, Tenorio AR, Montoya CJ, Gichinga CN, et al. (2007) Dendritic cells from HIV-1 infected individuals are less responsive to toll-like receptor (TLR) ligands. Cell Immunol 250: 75-84.

12. Sachdeva N, Asthana V, Brewer TH, Garcia D, Asthana D (2008) Impaired restoration of plasmacytoid dendritic cells in HIV-1-infected patients with poor CD4 $\mathrm{T}$ cell reconstitution is associated with decrease in capacity to produce IFN-alpha but not proinflammatory cytokines. J Immunol 181: 2887-2897.

13. Tilton JC, Manion MM, Luskin MR, Johnson AJ, Patamawenu AA, et al. (2008) Human immunodeficiency virus viremia induces plasmacytoid dendritic cell activation in vivo and diminished alpha interferon production in vitro. J Virol 82: 3997-4006.

14. Machmach K, Leal M, Gras C, Viciana P, Genebat M, et al. (2012) Plasmacytoid dendritic cells reduce HIV production in elite controllers. J Virol 86: 4245-4252.

15. Shang H, Xu J, Han X, Spero Li J, Arledge KC, et al. (2012) HIV prevention: Bring safe sex to China. Nature 485: 576-577.

16. O'Brien M, Manches O, Sabado RL, Baranda SJ, Wang Y, et al. (2011) Spatiotemporal trafficking of HIV in human plasmacytoid dendritic cells defines a persistently IFN-alpha-producing and partially matured phenotype. J Clin Invest 121: 1088-1101.

17. Guiducci C, Coffman RL, Barrat FJ (2009) Signalling pathways leading to IFNalpha production in human plasmacytoid dendritic cell and the possible use of agonists or antagonists of TLR7 and TLR9 in clinical indications. J Intern Med 265: 43-57.

18. Wu Z, Xu J, Liu E, Mao Y, Xiao Y, et al. (2013) HIV and syphilis prevalence among men who have sex with men: a cross-sectional survey of 61 cities in China. Clin Infect Dis 57: 298-309.

19. Liu YJ, Kanzler H, Soumelis V, Gilliet M (2001) Dendritic cell lineage, plasticity and cross-regulation. Nat Immunol 2: 585-589.

20. Manches O, Frleta D, Bhardwaj N (2014) Dendritic cells in progression and pathology of HIV infection. Trends Immunol 35: 114-122.

21. Martinelli E, Cicala C, Van Ryk D, Goode DJ, Macleod K, et al. (2007) HIV1 gp120 inhibits TLR9-mediated activation and IFN-\{alpha\} secretion in plasmacytoid dendritic cells. Proc Natl Acad Sci USA 104: 3396-3401.

22. Beignon AS, McKenna K, Skoberne M, Manches O, DaSilva I, et al. (2005) Endocytosis of HIV-1 activates plasmacytoid dendritic cells via Toll-like receptor-viral RNA interactions. J Clin Invest 115: 3265-3275.

23. Renshaw M, Rockwell J, Engleman C, Gewirtz A, Katz J, et al. (2002) Cutting edge: impaired Toll-like receptor expression and function in aging. J Immunol 169: 4697-4701.

24. Kaushik S, Teque F, Patel M, Fujimura SH, Schmidt B, et al. (2013) Plasmacytoid dendritic cell number and responses to Toll-like receptor 7 and 9 agonists vary in HIV Type 1-infected individuals in relation to clinical state. AIDS Res Hum Retroviruses 29: 501-510.

25. Xu N, Yao HP, Lv GC, Chen Z (2012) Downregulation of TLR7/9 leads to deficient production of IFN-Ît from plasmacytoid dendritic cells in chronic hepatitis B. Inflamm Res 61: 997-1004.

26. Honda K, Yanai H, Negishi H, Asagiri M, Sato M, et al. (2005) IRF-7 is the master regulator of type-I interferon-dependent immune responses. Nature 434: $772-777$. 